\title{
Growth performance of DZ-white and Improved Horro chicken breeds under different agro-ecological zones of Ethiopia
}

\author{
S. Mulugeta ${ }^{1 *}$, G. Goshu ${ }^{2}$, W. Esatu ${ }^{3}$
}

\begin{abstract}
${ }^{1}$ Department of Animal and Range Science, Wolaita Sodo University, P. O. Box 138, Wolaita Sodo, ${ }^{2}$ Department of Animal Production Studies, College of Veterinary Medicine and Agriculture, Addis Ababa University, ${ }^{3}$ International Livestock Research Institute (ILRI)P. Box 5689, Addis Ababa, Ethiopia,

*Corresponding author: samrijaf@yahoo.com, Tel: +251913200612
\end{abstract}

Journal of Livestock Science (ISSN online 2277-6214) 11: 45-53

Received on 2/12/2019; Accepted on 4/2/2020

doi. 10.33259/JLivestSci.2020.45-53

\begin{abstract}
The growth performances of DZ-white and Improved Horro chickens in three different agro-ecological zones were evaluated in the present study. 861 day-old unsexed chicks were randomly assigned to a $2 \times 3$ factorial arrangement in CRD involving two breeds (DZ-white and Improved Horro), three locations (Debre Berhan- L1, MelkaWererL2and Mekelle-L3 characterized as highland, lowland and midland respectively). FI, ADG, FCR. BW, BW gain and mortality were measured. Data were analyzed by GLM procedure of SAS .The result revealed that effect of breed on Initial BW,BW, BW gain, ADGFI was significantly $(\mathrm{P} \leq 0.05)$ higher and FCR was significantly $(\mathrm{P} \leq 0.05)$ better for DZ-white breed during the starter and grower phase. Among the locations significantly $(\mathrm{P} \leq 0.05)$ higher $\mathrm{BW}$ was recorded on L1 and L3 at both phases. During the starter phase significantly $(\mathrm{P} \leq 0.05)$ higher BW gain and ADG was recorded in the birds kept in L1 and L3. Birds consumed significantly $(\mathrm{P} \leq 0.05)$ more feed on L1 during the starter phase. However, significantly $(\mathrm{P} \leq 0.05)$ more feed were recorded on L1 and L3 during the grower phase and better FCR was recorded in L3 at both phases. Mortality was significantly $(\mathrm{P} \leq 0.05)$ higher on L2at both phases. There was a significant $(\mathrm{P} \leq 0.05)$ breed by location interaction for FI both at starter and grower phase and BW at grower phase. DZ-white exhibited the highest at L1 and L3, followed by DZ-white at L2 and Improved Horro at L3 and the lowest one was recorded for Improved Horro at L1 and L2.DZ-white was significantly $(\mathrm{P} \leq 0.05)$ superior in feed intake at L1 followed by Improved Horro at L1 and significantly $(\mathrm{P} \leq 0.05)$ lower feed intake was recorded in both breeds at L3 during the starter phase. However, DZ-white was significantly superior in feed intake at L1, followed by DZ-white at L3 and significantly $(\mathrm{P} \leq 0.05)$ lower feed intake was recorded in the Improved Horro across the location and DZ-white at L2 during the grower phase. In conclusion, the results of this study have demonstrated that DZ-white breeds had significantly enhanced speed of weight gain as well as growth and good feed conversion ratio. However, this study has also shown that Improved Horro chickens breeds have the genetic ability to grow fast if properly managed. Both chicken breeds are well adapted in highland and midland agro-ecology zone and their production performance was better than the chicken kept in lowland agro-ecology zone.
\end{abstract}

Keywords: Growth performance; Improved Horro; DZ-white; poultry; Agro-ecological zones of Ethiopia 


\section{Introduction}

Poultry has been increasingly recognized as one of the entry points to address the problems of malnutrition, food insecurity, low income and poverty as a whole. In fact, Dei et al. (2009) argue that poultry is a profitable venture and eventually a tool for livelihood improvement and poverty alleviation in the developing countries (Sharma, 2007; Ekka et al, 2016). Moreover, it occupies a unique position in terms of its contribution to the provision of high quality protein food to rural smallholder farming families in Africa (Sonaiya et al., 1999), Asia and particularly in Ethiopia (Tadelle and Ogle, 2001).

Although the sector have been contributing substantially to household income and nutrition for majority of poor rural communities (Alabi et al., 2006), its expansion has been limited by low productivity. Poor management practices, high prevalence of diseases and low genetic potential of the stock have been the main factors associated with low productivity of the sector (Lwelamira, 2007). Therefore, for improving performance of local chickens and productivity of the sector, interventions on genetic potential improvement through appropriate breeding programs and defining production environments were imperative. Consequently; this will increase the genetic potential for both eggs and meat and make a substantial contribution to reduce poverty and malnutrition among rural and urban poor (LMP, 2015).

In Ethiopia as an alternative approach to improving production levels, a breeding program of indigenous chicken was initiated in 2008 using chicken from Horro region; with objectives of improving village chicken productivity through selective breeding for higher productivity and a more optimal adaptive capacity to withstand harsh condition than unselected population (Nigussie et al., 2010). The breeding program successfully increased average body weight at 16 weeks by about $74 \%$ and egg number of 24 weeks after on-set of egg lying by $21 \%$ from generation 4 to 6 (Wondmeneh, 2015). In parallel to Horro breeding program in 2009 DZARC developed a synthetic breed known as DZ-white. DZ-white has been synthesized from Lohman silver, Koekoek and Rhode Island White (RIW) line. The main purpose of crossing DZ-white chickens was to develop breeds for the semi intensive or the substandard production system for contributing to the improvement of living conditions of Ethiopian women and youth farmers.

The work presented here is part of an on-going project to produce a synthetic chicken population through cross breeding scheme. The improved Horro and DZ-white obtained in the breeding program has made tremendous progress within ongoing evaluations made at DZARC for 10 (Improved Horro) and 5 (DZ-white) generations. However, growth performance of DZ-white has never been evaluated at different agro ecological zones of the country and also the growth performance of Improved Horro has limited information in different agro-ecological zones.it was found important to evaluate the growth performance of these breeds in different agro-ecological zones of the country before they are released to the end users. Therefore, the objective of this study was to evaluate the growth performances of DZ-white and Improved Horro chickens in three different agro-ecological zones of Ethiopia.

\section{Materials and Methods Description of the Study Area}

The study was conducted in three different agro-ecological zones of Ethiopia; Debre Berhan(faji temperate fruits and related farm), Mekelle (Mekelle University)and Melka Werer (Werer Agricultural Research Center)which were characterized as highland, midland, and lowland, respectively.

Debre Berhan is located at the distance of $120 \mathrm{~km}$ North East of Addis Ababa. The area is located at latitude of $9^{\circ} 36^{`} \mathrm{~N}$ and $39^{\circ} 38^{`} \mathrm{E}$ longitude; it is situated at an altitude of 2828 meters above sea level. The mean annual rainfall of the area ranges from 781 to $1279 \mathrm{~mm}$. The maximum and minimum temperature is $24^{\circ} \mathrm{c}$ and $6.1^{\circ} \mathrm{Crespectively}$. It has sub moist humidity $62.3 \%$ (EIAR, 2004).

Mekelle is located at the distance of $783 \mathrm{Km}$ North East of Addis Ababa. The area is located at $39^{\circ} 29^{\circ} \mathrm{E}$ and $13^{\circ}$ $30^{\circ} \mathrm{N}$ at an altitude of 2000 meters above sea level. The climate is semi-arid with a mean annual rainfall of $619 \mathrm{~mm}$ and the annual maximum and minimum temperature is about $29.9^{\circ} \mathrm{C}$ and $11.8^{\circ} \mathrm{C}$, respectively (BoPED, 2011).

Melka Werer is located in the Southern part of the Afar National Regional State, about $280 \mathrm{~km}$ east of Addis Ababa. The geographical location of the study area is between $9^{\circ} 16^{\prime} \mathrm{N}$, longitude $40^{\circ} 9^{\prime} \mathrm{E}$ and at an altitude of 750 meters above sea level. The climate is typically semi-arid with annual average rainfall $590 \mathrm{~mm}$. Its average monthly temperature is about $26.7{ }^{\circ} \mathrm{C}$ with maximum temperature of $40.8^{\circ} \mathrm{C}$ and minimum $26.7^{\circ} \mathrm{C}$ (EIAR, 1988). 


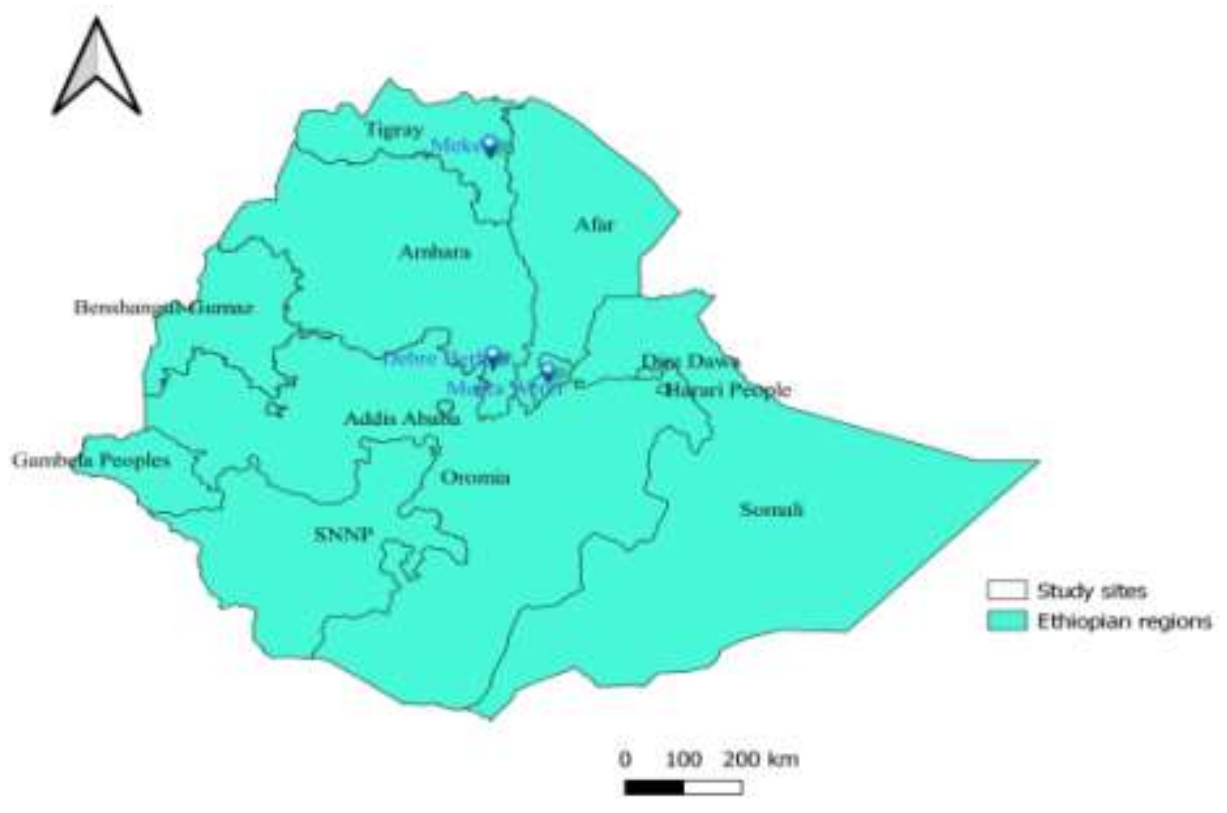

Fig1. Location Map of Study area.

\section{Experimental Breeds}

The chicken breeds used in the study were Improved Horro and DZ-white (synthetic breed from Lohman silver, KK and RIW). The predominant plumage color for male and female Improved Horro chicken is a reddish brown or medium to light brown. On the other hand the predominant plumage color of DZ-white for both males and female is completely white. In this study a total of eight hundred sixteen one day-old chicks (both sex) were used (Table 1).

\section{Experimental Design}

The experiment was arranged in $2 \times 3$ factorial experiments in Completely Randomized Design (CRD) involving two breeds (DZ-white and Improved Horro) and three locations (Debre Berhan- $\mathrm{L}_{1}$, MelkaWerer- $\mathrm{L}_{2}$ and Mekelle- $\mathrm{L}_{3}$.

\section{Housing and management}

Eggs were hatched using the hatchery units of the poultry division at DZARC following standard procedures of the pass reform hatchery machines. After hatching, each chick was vaccinated against Marek's disease with HVT strain, weighed and randomly allocated to the chick box and transported to their experimental location. At the time they reached their experimental location, the chicks were randomly allocated to experimental pens in corrugated iron house with partial open-side which has a wire net on the open sides. Each location had 6 separate pens of an equal size, each pens were installed using mesh wire partitions. They were placed in deep litter pens, using a teff (Eragrostis teff) straw as litter material and light was provided according to Bovans browns breed management guide for 6 weeks. All experimental chickens were fed with starter rations (Table 2) for a period of 0-8 weeks and a grower ration for period of 9-16 weeks. Feed and water was provided ad libitum during the brooding and growth phases. The experimental chicks were vaccinated against Newcastle disease on day 5 with HB1 and on day 28 and 60 with Lasota strain; against Infectious Bursal Disease on day 7 and 21; with gumboro vaccine and against Fowl typhoid on day 45. Antibiotics and vitamins were supplied for all chicken flocks under study when disease was suspected in a pen. The same raring and vaccination were followed throughout the experimental period for all locations. 


\section{Data Collection \\ Body weight measurements}

Body weight of the chicks were taken at the beginning and recorded as initial weight. Then, the average body weight was measured every 7 days using a sensitive balance by weighing chicks in each pen. The overall average body weight for each treatment was then computed by taking an average values for the replication.

Table 1. Day-old chick's distribution among locations.

\begin{tabular}{|c|c|c|}
\hline \multirow[t]{2}{*}{ Location } & \multicolumn{2}{|c|}{ Breed } \\
\hline & DZ-white & Improved Horro \\
\hline Debre Berhan & 142 & 163 \\
\hline Melka Werer & 145 & 150 \\
\hline Mekelle & 136 & 125 \\
\hline
\end{tabular}

Table 2. Proportion of ingredients and their respective gross composition of diets.

\begin{tabular}{|l|l|l|}
\hline Ingredients (\%) & Starter(0-8) & Grower(9-16) \\
\hline white maize & 58 & 60 \\
\hline wheat middling & 8 & 12.57 \\
\hline SBM & 25.17 & 18.62 \\
\hline NSC & 5 & 5 \\
\hline Salt & 0.4 & 0.4 \\
\hline Limestone & 2.48 & 2.58 \\
\hline Premix & 0.5 & 0.5 \\
\hline DL-lysine & 0.25 & 0.3 \\
\hline DL-methionine & 0.2 & 0.03 \\
\hline Composition (\% DM basis) & & \\
\hline DM & 88.88 & 89.130 \\
\hline ME(kcal/kg ) & 2850 & 2800 \\
\hline CP & 28.941 & 23.089 \\
\hline Cfat & 5.009 & 1.066 \\
\hline CF & 3.319 & 2.031 \\
\hline Ca & 3.375 & 2.244 \\
\hline P & 0.5 & 0.5 \\
\hline
\end{tabular}

\section{Feed intake and feed conversion ratio (FCR)}

A weighed amount of feed was offered once daily at 08:00 am every day and the refuse was collected next morning every day and weighed after removing external contaminants by visual inspection and hand picking. The feed offer and refuse were recorded for each replicate. Feed conversion ratio (FCR) was measured dividing the feed consumed by the live weight gain within consecutive weeks.

Mortality

Mortality was recorded daily as encountered throughout the experimental period and expressed as percent mortality at the end of the experiment.

\section{Statistical Analysis}

Growth data were analyzed by General Linear Model's procedures of Statistical Analysis System (SAS) version 9.3 (SAS, 2010).

$$
\mathrm{Y}_{\mathrm{ijk}}=\mu+\alpha_{\mathrm{i}}+\beta_{\mathrm{j}}+(\alpha \beta)_{\mathrm{ij}}+\mathrm{e}_{\mathrm{ijk}}
$$

Where: $\mathrm{Y}_{i j k}=$ the dependent variables for the $\mathrm{i}^{\text {th }}$ and $\mathrm{j}^{\text {th }}$ observation $\mu=$ overall mean effect, $\alpha_{i}=$ the $i^{\text {th }}$ effect of two breed effect

$\beta_{j}=$ is the $j^{\text {th }}$ effect of the three environment; $(\alpha \beta)_{\mathrm{ij}}=$ interaction of main effect

$\mathrm{e}_{i j k}=$ the random error variation

\section{Results}

The initial BW of chicks at day-old showed highly significant difference $(\mathrm{P} \leq 0.001)$ between breeds. DZwhite were significantly $(\mathrm{P} \leq 0.05)$ higher and significantly $(\mathrm{P} \leq 0.05)$ lower initial $\mathrm{BW}$ was recorded in Improved Horro breed. Location had no significant effect $(\mathrm{P} \geq 0.05)$ on initial BW (Table 3). On the other hand the interaction between breed and location (Table 4 ) showed nearly similar with no significant differences $(\mathrm{P} \geq 0.05)$. 
The effect of breed and locations on BW (BW8 and BW16), BW gain and ADG of chicks during the starter and grower phase are shown in (Table 3 and 5). The BW (BW8 and BW16), showed highly significant difference $(\mathrm{P} \leq 0.001)$ between breeds, the DZ-white exhibit the highest BW8 (540.78g) and BW16 (1433.07g) and the lowest BW8 (469.55g) and BW16 (1179.87g) was recorded in Improved Horro. The main effect location revealed that the BW8 and BW16 for the birds kept in L1 and L3 was highest $(\mathrm{P} \leq 0.05)$ as compared to those birds kept in L2. On the other hand, BW8 showed values with no significant differences $(\mathrm{P} \geq 0.05)$ between interaction effects of breed and location (Table 4). However a significant $(\mathrm{P} \leq 0.05)$ difference noted between the two-way interaction effects of breed and location on BW16, DZ-white exhibited the highest at L1 and L3, followed by DZ-white at L2 and Improved Horro at L3 and the lowest one was recorded for Improved Horro at L1 and L2 (Table 6).

The BW gain and ADG for DZ-white was highest $(\mathrm{P} \leq 0.001)$ as compared to Improved Horro both at starter and grower phase. From location main effect, BW gain and ADG was significantly ( $\mathrm{P} \leq 0.05)$ higher in L1 and L3 and significantly $(\mathrm{P} \leq 0.05)$ lower in L2 during the starter phase (Table 3). However, BW gain and ADG showed values with no significant differences during the grower phase (Table 5). On breed by location interaction (Table 4 and 6) $\mathrm{BW}$ gain and $\mathrm{ADG}$ showed values with no significant differences $(\mathrm{P} \geq 0.05)$ both at starter and grower phase.

As shown in (Table 3 and 5), significantly $(\mathrm{P} \leq 0.05)$ higher feed intake was observed in DZ-white compared to the Improved Horro during the starter and grower phase. There was also a significant $(\mathrm{P} \leq 0.05)$ difference between locations. Birds consumed significantly $(\mathrm{P} \leq 0.05)$ more on $\mathrm{L} 1$ followed by $\mathrm{L} 2$ and consumed low amount $(\mathrm{P} \leq 0.05)$ on L3 during the starter phase. However, birds consumed significantly $(\mathrm{P} \leq 0.05)$ more on L1 and L3 and consumed low amount $(\mathrm{P} \leq 0.05)$ on L2 during the grower phase. The effect of breed by location interaction (Table 4) on the other hand, indicated that DZ-white was significantly $(\mathrm{P} \leq 0.05)$ superior in feed intake at L1 followed by Improved Horro at L1 and significantly $(\mathrm{P} \leq 0.05)$ lower feed intake was recorded in both breeds atL3 during the starter phase. However, DZ-white was significantly superior in feed intake at L1, followed by DZ-white at L3and significantly $(\mathrm{P} \leq 0.05)$ lower feed intake was recorded in the Improved Horro across the location and DZ-white at L2 during the grower phase (Table 6).

As shown in (Table 3 and 5), the main effect of breed showed that DZ-white had significantly $(\mathrm{P} \leq 0.05)$ improved feed conversion ratio (FCR) over Improved Horro during the starter and grower phase. The main effect location revealed that significantly $(\mathrm{P} \leq 0.001)$ higher FCR was observed in birds kept at L3 as compared to birds kept at L1 and L2 during the starter phase (Table 3). However significantly $(\mathrm{p}<0.5)$ higher FCR was recorded in L3 and significantly lower FCR was observed in L2 during the grower phase (Table 5). From the interaction effect, FCR showed values with no significant differences $(\mathrm{P} \geq 0.05)$ between the two-way interaction effects of breed and location (Table 4 and 6).

In this study, there was no significant $(\mathrm{P} \geq 0.05)$ difference on breed main effect for mortality during the starter and grower phase. From location main effect, significantly $(\mathrm{P} \leq 0.05)$ higher mortality was recorded in L2 as compared to L1 and L3 due to unidentified diseases (UD). The higher values due to UD were $10.78 \%$ and $17.66 \%$ during starter and grower phase respectively (Table 3 and 5). However, there was no significant difference ( $\mathrm{P} \geq 0.05)$ between interaction effects of breed and location both at starter and grower phase (Table 4 and 6 ).

Table 3: LSM and SEM values for the main effects of breed and location on growth traits during the starter phase.

\begin{tabular}{|c|c|c|c|c|c|c|c|}
\hline \multirow{2}{*}{$\begin{array}{l}\text { Parameter } \\
\text { (g/bird) }\end{array}$} & \multicolumn{3}{|c|}{ Breed } & \multicolumn{4}{|c|}{ Location } \\
\hline & DZ-white & $\begin{array}{l}\text { Improved } \\
\text { Horro }\end{array}$ & $\mathrm{P}$ value & $\mathrm{L}_{1}$ & $\mathrm{~L}_{2}$ & $\mathrm{~L}_{3}$ & $\mathrm{P}$ value \\
\hline Initial BW & $\begin{array}{l}34.96^{\mathrm{a}} \\
\pm 0.10\end{array}$ & $\begin{array}{l}29.27^{b} \\
\pm 0.18\end{array}$ & 0.0001 & $\begin{array}{l}32.36 \\
\pm 1.17\end{array}$ & $\begin{array}{l}31.92 \\
\pm 1.42\end{array}$ & $\begin{array}{l}32.10 \\
\pm 1.26\end{array}$ & 0.1540 \\
\hline BW8 & $\begin{array}{l}540.78^{a} \\
\pm 10.84\end{array}$ & $\begin{array}{l}469.55^{\mathrm{b}} \\
\pm 10.47\end{array}$ & 0.0001 & $\begin{array}{l}521.79^{\mathrm{a}} \\
\pm 21.45\end{array}$ & $\begin{array}{l}473.00^{b} \\
\pm 16.23\end{array}$ & $\begin{array}{l}520.71^{\mathrm{a}} \\
\pm 16.49\end{array}$ & 0.0046 \\
\hline BW gain & $\begin{array}{l}505.80^{\mathrm{a}} \\
\pm 10.84\end{array}$ & $\begin{array}{l}440.28^{\mathrm{b}} \\
\pm 10.33\end{array}$ & 0.0001 & $\begin{array}{l}489.43 \\
\pm^{\mathrm{a}} 20.39\end{array}$ & $\begin{array}{l}441.08^{\mathrm{b}} \\
\pm 15.08\end{array}$ & $\begin{array}{l}488.61^{\mathrm{a}} \\
\pm 15.24\end{array}$ & 0.0045 \\
\hline ADG (g/b/d) & $\begin{array}{l}9.03^{\mathrm{a}} \\
\pm 0.19\end{array}$ & $\begin{array}{l}7.86^{\mathrm{b}} \\
\pm 0.18\end{array}$ & 0.0001 & $\begin{array}{l}8.74^{\mathrm{a}} \\
\pm 0.36\end{array}$ & $\begin{array}{l}7.88^{\mathrm{b}} \\
\pm 0.27\end{array}$ & $\begin{array}{l}8.73^{\mathrm{a}} \\
\pm 0.27\end{array}$ & 0.0045 \\
\hline Feed intake & $\begin{array}{l}1732.80^{\mathrm{a}} \\
\pm 114.46\end{array}$ & $\begin{array}{l}1637.39^{b} \\
\pm 77.45\end{array}$ & 0.0004 & $\begin{array}{l}1994.42^{\mathrm{a}} \\
\pm 58.60\end{array}$ & $\begin{array}{l}1720.15^{b} \\
\pm 19.19\end{array}$ & $\begin{array}{l}1340.74^{\mathrm{c}} \\
\pm 17.47\end{array}$ & 0.0001 \\
\hline FCR & $\begin{array}{l}3.21^{\mathrm{b}} \\
\pm 0.21\end{array}$ & $\begin{array}{l}3.51^{\mathrm{a}} \\
\pm 0.20\end{array}$ & 0.0130 & $\begin{array}{l}3.84^{\mathrm{a}} \\
\pm 0.10\end{array}$ & $\begin{array}{l}3.66^{\mathrm{a}} \\
\pm 0.12\end{array}$ & $\begin{array}{l}2.59^{\mathrm{b}} \\
\pm 0.09\end{array}$ & 0.0001 \\
\hline $\begin{array}{l}\text { Mortality } \\
(\%)\end{array}$ & $\begin{array}{l}7.36 \\
\pm 2.35\end{array}$ & $\begin{array}{l}4.69 \\
\pm 1.14\end{array}$ & 0.2301 & $\begin{array}{l}5.02^{\mathrm{b}} \\
\pm 0.76\end{array}$ & $\begin{array}{l}10.78^{\mathrm{a}} \\
\pm 2.83\end{array}$ & $\begin{array}{l}2.28^{\mathrm{b}} \\
\pm 1.15\end{array}$ & 0.0187 \\
\hline
\end{tabular}

*a-c LSM \pm SEM within a row with different superscripts differ significantly $(\mathrm{P} \geq 0.05), \mathrm{L}=$ location, $\mathrm{L}_{1}=\mathrm{DebreBerhan}, \mathrm{L}_{2}=$ MelkaWerer, $\mathrm{L}_{3}=$ Mekelle, $\mathrm{BW}=$ Body weight, $\mathrm{BW} 8=\mathrm{BW}$ at week $8, \mathrm{ADG}=$ average daily gain, $\mathrm{FCR}=\mathrm{Feed}$ conversion ratio, $\mathrm{LSM}=$ least squares mean, $\mathrm{SEM}=$ Standard error of the mean, $\mathrm{P}=$ Probability. 
Mulugeta et al, 2020/ J. Livestock Sci. 11: 45-53

Table 4: LSM and SEM values for the interaction effects of breed and location on growth traits during the starter phase.

\begin{tabular}{|c|c|c|c|c|c|c|c|c|c|}
\hline \multicolumn{3}{|c|}{ 2-way interactions } & Initial BW & BW8 & BW gain & ADG & Feed intake & FCR & Mortality (\%) \\
\hline \multirow{6}{*}{ Br*L } & \multirow{3}{*}{ DZ-white } & L1 & $\begin{array}{l}34.95 \\
\pm 0.19\end{array}$ & $\begin{array}{l}564.21 \\
\pm 12.12\end{array}$ & $\begin{array}{l}529.26 \\
\pm 12.11\end{array}$ & $\begin{array}{l}9.45 \\
\pm 0.22\end{array}$ & $\begin{array}{l}2123.25^{\mathrm{a}} \\
\pm 14.72\end{array}$ & $\begin{array}{l}3.77 \\
\pm 0.10\end{array}$ & $\begin{array}{l}6.34 \\
\pm 0.04\end{array}$ \\
\hline & & L2 & $\begin{array}{l}35.09 \\
\pm 0.24\end{array}$ & $\begin{array}{l}501.33 \\
\pm 6.67\end{array}$ & $\begin{array}{l}466.25 \\
\pm 6.47\end{array}$ & $\begin{array}{l}8.33 \\
\pm 0.12\end{array}$ & $\begin{array}{l}1739.72^{\mathrm{c}} \\
\pm 21.57\end{array}$ & $\begin{array}{l}3.47 \\
\pm 0.08\end{array}$ & $\begin{array}{l}13.56 \\
\pm 5.31\end{array}$ \\
\hline & & L3 & $\begin{array}{l}34.92 \\
\pm 0.17\end{array}$ & $\begin{array}{l}556.81 \\
\pm 6.07\end{array}$ & $\begin{array}{l}521.89 \\
\pm 5.91\end{array}$ & $\begin{array}{l}9.32 \\
\pm 0.11\end{array}$ & $\begin{array}{l}1335.44^{\mathrm{d}} \\
\pm 36.51\end{array}$ & $\begin{array}{l}2.40 \\
\pm 0.09\end{array}$ & $\begin{array}{l}2.17 \\
\pm 2.17\end{array}$ \\
\hline & \multirow[t]{4}{*}{$\begin{array}{l}\text { Improved } \\
\text { Horro }\end{array}$} & L1 & $\begin{array}{l}29.77 \\
\pm 0.29 \\
\end{array}$ & $\begin{array}{l}479.36 \\
\pm 18.82 \\
\end{array}$ & $\begin{array}{l}449.59 \\
\pm 18.60 \\
\end{array}$ & $\begin{array}{l}8.03 \\
\pm 0.33 \\
\end{array}$ & $\begin{array}{l}1865.58^{\mathrm{b}} \\
\pm 18.77 \\
\end{array}$ & $\begin{array}{l}3.91 \\
\pm 0.20\end{array}$ & $\begin{array}{l}3.69 \\
\pm 1.08 \\
\end{array}$ \\
\hline & & L2 & $\begin{array}{l}28.75 \\
\pm 0.22\end{array}$ & $\begin{array}{l}444.67 \\
\pm 21.67\end{array}$ & $\begin{array}{l}415.91 \\
\pm 21.48\end{array}$ & $\begin{array}{l}7.43 \\
\pm 0.38\end{array}$ & $\begin{array}{l}1700.58^{\mathrm{c}} \\
\pm 31.51\end{array}$ & $\begin{array}{l}3.84 \\
\pm 0.17\end{array}$ & $\begin{array}{l}8.00 \\
\pm 2.00\end{array}$ \\
\hline & & L3 & $\begin{array}{l}29.28 \\
\pm 0.10\end{array}$ & $\begin{array}{l}484.61 \\
\pm 4.40\end{array}$ & $\begin{array}{l}455.33 \\
\pm 4.38\end{array}$ & $\begin{array}{l}8.13 \\
\pm 0.08\end{array}$ & $\begin{array}{l}1346.03^{\mathrm{d}} \\
\pm 12.84\end{array}$ & $\begin{array}{l}2.78 \\
\pm 0.00\end{array}$ & $\begin{array}{l}2.38 \\
\pm 1.37\end{array}$ \\
\hline P value & & & 0.0517 & 0.5866 & 0.5557 & 0.5557 & 0.0003 & 0.5696 & 0.5528 \\
\hline
\end{tabular}

$* \mathrm{a}-\mathrm{c} \mathrm{LSM} \pm \mathrm{SEM}$ within a row with different superscripts differ significantly $(\mathrm{P} \geq 0.05), \mathrm{Br} * \mathrm{~L}=$ Breed by location interaction, $\mathrm{L}=$ location, $\mathrm{L} 1$ $=$ DebreBerhan, $\mathrm{L} 2=$ MelkaWerer, $\mathrm{L} 3=$ Mekelle, $\mathrm{BW}=$ Body weight, $\mathrm{BW} 8=\mathrm{BW}$ at week $8, \mathrm{ADG}=$ average daily gain, $\mathrm{FCR}=\mathrm{Feed}$ conversion ratio, $\mathrm{LSM}=$ least squares mean, $\mathrm{SEM}=$ Standard error of the mean, $\mathrm{P}=$ Probability.

Table 5: LSM and SEM values for the main effects of breed and location on growth traits during the grower phase.

\begin{tabular}{|c|c|c|c|c|c|c|c|}
\hline \multirow{2}{*}{$\begin{array}{l}\text { Parameter } \\
\text { (g/bird) }\end{array}$} & \multicolumn{3}{|c|}{ Breed } & \multicolumn{4}{|c|}{ Location } \\
\hline & DZ-white & $\begin{array}{l}\text { Improved } \\
\text { Horro }\end{array}$ & $P$ value & L1 & L2 & L3 & $\mathrm{P}$ value \\
\hline BW16 & $\begin{array}{l}1372.47^{\mathrm{a}} \\
\pm 25.15\end{array}$ & $\begin{array}{l}1179.87^{\mathrm{b}} \\
\pm 17.64\end{array}$ & 0.0001 & $\begin{array}{l}1302.27^{\mathrm{a}} \\
\pm 59.47\end{array}$ & $\begin{array}{l}1207.85^{b} \\
\pm 38.07\end{array}$ & $\begin{array}{l}1318.40^{\mathrm{a}} \\
\pm 37.64\end{array}$ & 0.0003 \\
\hline $\begin{array}{l}\text { BW } \\
\text { gain }\end{array}$ & $\begin{array}{l}831.69^{\mathrm{a}} \\
\pm 16.63\end{array}$ & $\begin{array}{l}710.33^{b} \\
\pm 17.48\end{array}$ & 0.0001 & $\begin{array}{l}780.48 \\
\pm 42.51\end{array}$ & $\begin{array}{l}734.85 \\
\pm 29.78\end{array}$ & $\begin{array}{l}797.69 \\
\pm 22.15\end{array}$ & 0.0672 \\
\hline $\begin{array}{l}\text { ADG } \\
(\mathrm{g} / \mathrm{b} / \mathrm{d})\end{array}$ & $\begin{array}{l}14.85^{\mathrm{a}} \\
\pm 0.30\end{array}$ & $\begin{array}{l}12.68^{\mathrm{b}} \\
\pm 0.31\end{array}$ & 0.0001 & $\begin{array}{l}13.94 \\
\pm 0.76\end{array}$ & $\begin{array}{l}13.12 \\
\pm 0.53\end{array}$ & $\begin{array}{l}14.24 \\
\pm 0.40\end{array}$ & 0.0672 \\
\hline $\begin{array}{l}\text { Feed } \\
\text { intake }\end{array}$ & $\begin{array}{l}3943.10^{\text {a }} \\
\pm 60.18\end{array}$ & $\begin{array}{l}3746.10^{b} \\
\pm 21.20\end{array}$ & 0.0001 & $\begin{array}{l}3908.21^{\mathrm{a}} \\
\pm 95.11\end{array}$ & $\begin{array}{l}3727.53^{b} \\
\pm 27.17\end{array}$ & $\begin{array}{l}3898.06^{\mathrm{a}} \\
\pm 41.07\end{array}$ & 0.0006 \\
\hline FCR & $\begin{array}{l}2.87^{\mathrm{b}} \\
\pm 0.02\end{array}$ & $\begin{array}{l}3.18^{\mathrm{a}} \\
\pm 0.04\end{array}$ & 0.0001 & $\begin{array}{l}3.02^{\mathrm{ab}} \\
\pm 0.07\end{array}$ & $\begin{array}{l}3.10^{\mathrm{a}} \\
\pm 0.09\end{array}$ & $\begin{array}{l}2.96^{\mathrm{b}} \\
\pm 0.06\end{array}$ & 0.0470 \\
\hline $\begin{array}{l}\text { Mortality } \\
(\%)\end{array}$ & $\begin{array}{l}5.42 \\
\pm 2.47\end{array}$ & $\begin{array}{l}9.52 \\
\pm 4.17\end{array}$ & 0.2645 & $\begin{array}{l}2.37^{b} \\
\pm 1.25\end{array}$ & $\begin{array}{l}17.66^{\mathrm{a}} \\
\pm 5.03\end{array}$ & $\begin{array}{l}2.38^{b} \\
\pm 0.85\end{array}$ & 0.0051 \\
\hline
\end{tabular}

*a-c LSM \pm SEM within a row with different superscripts differ significantly $(\mathrm{P} \geq 0.05), \mathrm{L}=$ location, $\mathrm{L} 1=$ DebreBerhan, $\mathrm{L} 2=\mathrm{MelkaWerer}, \mathrm{L} 3=$ Mekelle, $\mathrm{BW}=$ Body weight, $\mathrm{BW} 16=\mathrm{BW}$ at week $16, \mathrm{ADG}=$ average daily gain, $\mathrm{FCR}=$ Feed conversion ratio, $\mathrm{LSM}=$ least squares mean, $\mathrm{SEM}=$ Standard error of the mean, $\mathrm{P}=$ Probability

Table 6: LSM and SEM values for the interaction effects of breed and location on growth traits during the grower phase.

\begin{tabular}{|c|c|c|c|c|c|c|c|c|}
\hline \multicolumn{3}{|c|}{ 2-way interactions } & BW16 & BW gain & ADG & Feed & FCR & Mortality \\
\hline \multirow{6}{*}{$\mathbf{B r} * \mathbf{L}$} & \multirow{3}{*}{ DZ-white } & L1 & $\begin{array}{l}1433.07 a \\
\pm 10.39\end{array}$ & $\begin{array}{l}868.86 \\
\pm 17.65\end{array}$ & $\begin{array}{l}15.52 \\
\pm 0.32\end{array}$ & $\begin{array}{l}4112.25 \mathrm{a} \\
\pm 57.31\end{array}$ & $\begin{array}{l}2.87 \\
\pm 0.04\end{array}$ & $\begin{array}{l}2.27 \\
\pm 1.31\end{array}$ \\
\hline & & L2 & $\begin{array}{l}1285.75 b \\
\pm 30.37\end{array}$ & $\begin{array}{l}784.42 \\
\pm 26.99\end{array}$ & $\begin{array}{l}14.01 \\
\pm 0.48\end{array}$ & $\begin{array}{l}3733.65 \mathrm{c} \\
\pm 51.89\end{array}$ & $\begin{array}{l}2.91 \\
\pm 0.03\end{array}$ & $\begin{array}{l}12.50 \\
\pm 5.59\end{array}$ \\
\hline & & L3 & $\begin{array}{l}1398.59 \mathrm{a} \\
\pm 24.98\end{array}$ & $\begin{array}{l}841.79 \\
\pm 20.47\end{array}$ & $\begin{array}{l}15.03 \\
\pm 0.37\end{array}$ & $\begin{array}{l}3983.40 b \\
\pm 20.87\end{array}$ & $\begin{array}{l}2.85 \\
\pm 0.06\end{array}$ & $\begin{array}{l}1.48 \\
\pm 1.48\end{array}$ \\
\hline & \multirow{3}{*}{$\begin{array}{l}\text { Improved } \\
\text { Horro }\end{array}$} & L1 & $\begin{array}{l}1171.47 \mathrm{c} \\
\pm 21.63\end{array}$ & $\begin{array}{l}692.11 \\
\pm 30.25\end{array}$ & $\begin{array}{l}12.36 \\
\pm 0.54\end{array}$ & $\begin{array}{l}3704.16 \mathrm{c} \\
\pm 17.59\end{array}$ & $\begin{array}{l}3.16 \\
\pm 0.05\end{array}$ & $\begin{array}{l}2.47 \\
\pm 2.47\end{array}$ \\
\hline & & $\mathrm{L} 2$ & $\begin{array}{l}1129.94 \mathrm{c} \\
\pm 16.02\end{array}$ & $\begin{array}{l}685.28 \\
\pm 35.32\end{array}$ & $\begin{array}{l}12.24 \\
\pm 0.63\end{array}$ & $\begin{array}{l}3721.41 \mathrm{c} \\
\pm 30.99\end{array}$ & $\begin{array}{l}3.30 \\
\pm 0.07\end{array}$ & $\begin{array}{l}22.82 \\
\pm 8.29\end{array}$ \\
\hline & & L3 & $\begin{array}{l}1238.21 \mathrm{~b} \\
\pm 5.21\end{array}$ & $\begin{array}{l}753.60 \\
\pm 9.47\end{array}$ & $\begin{array}{l}13.46 \\
\pm 0.17\end{array}$ & $\begin{array}{l}3812.73 \mathrm{c} \\
\pm 26.79\end{array}$ & $\begin{array}{l}3.08 \\
\pm 0.03\end{array}$ & $\begin{array}{l}3.27 \\
\pm 0.80\end{array}$ \\
\hline$P$ value & & & 0.0356 & 0.1943 & 0.1943 & 0.0007 & 0.2848 & 0.47 \\
\hline
\end{tabular}

$* a-c$ LSM $\pm S E M$ within a row with different superscripts differ significantly $(P \geq 0.05), B r^{*} L=$ Breed by location interaction, $L=$ location, $L 1$ $=$ DebreBerhan, $L 2=$ MelkaWerer, $L 3=$ Mekelle, $B W=$ Body weight, $B W 16=B W$ at week $16, A D G=$ average daily gain, FCR $=F e e d$ conversion ratio, $L S M=$ least squares mean, $S E M=$ Standard error of the mean, $P=$ Probability 
Mulugeta et al, 2020/ J. Livestock Sci. 11: 45-53

\section{Discussion}

Body weight is the direct reflection of growth and it influences the production and reproduction traits of birds. In the present study the results on body weight at eight and sixteen weeks of age were significantly different in the two breeds. The significant genotype differences in body weight between the chicken showed that this trait is highly influenced by genetic factors and this agrees with reports of several authors (Mohammed et al., 2005; Adedeji, et al., 2006). Wondmeneh, (2015) also recorded differences in body weights among different chicken breeds from 8 to 20 weeks of age. DZ-white chicken tended to record higher weights than the Improved Horro counterparts during the starter and grower phase, the mean average weight recorded for the unsexed DZ-white chicken at 16 week rearing period was about $192.6 \mathrm{~g}$ higher $(\mathrm{P}<0.05)$ than in unsexed Improved Horro chicken. Wondmeneh, (2015)reported body weights of $428.9 \pm 0.9$ at 8 weeks and $873.4 \pm 1.9$ at 16 weeks in Horro chicken which were lower than the present estimates. The body weights of DZ-white at 16 weeks were comparable with the Potchefstroom koekoek which was 1399-1700.71g at 20 weeks of age (Wondmeneh et al., 2012), On another hand the observed body weights in DZ-white in this studies was higher than the one reported by Wondmeneh, (2015) for Bovan brown at 16 weeks of age under on station management.

The growth performance of chicken is mostly affected by the environment, which reflects the differences in geographic location. In this study Chickens in L1 and L3 had higher body weight than those in L2; this is because of the difference weather among the locations. Similar to the present study the result of Alem (2014) reported that, the average weight of mature males (cocks) was significantly higher $(\mathrm{P}<0.05)$ in midland than in lowland agro-ecology in Ethiopia. Regarding the interaction effects, during the growth phase similar to the present study the result of Kayitesi, $(2015)$ reported Kuroiler chickens reared in Gulu district were significantly heavier $(\mathrm{P}<0.05)$ than those Kuroiler chickens reared in Kiryandongo district.

Breeds of chickens have different genetic potential for growth and this explains why DZ-white chicken had faster rate of growth as compared to Improved Horro during the starter and grower phase. These results suggest that the lower chick weight and weight gain in Improved Horro might be attributable to breed differences. Correspondingly, this has also been shown by Nigussie et al. (2011) who reported that the growth of chickens is moderately dependent on genetics of the chickens. Reta et al. (2012) also got different performance between two breeds of chicken in Ethiopia. Moreover, differences in weight gain among different breeds and strains reported (Enaiat et al., 2010; Bekele et al., 2010; Ewonetu, 2017). The growth performance of chicken in L1 and L3 performed better than those in L2 during the starter phase. The results of the present study agreed with those of Mutayoba et al. (2012)which showed that growth of local chickens in Tanzania significantly differed $(\mathrm{P}<0.05)$ between locations. However, there was no variation in BW gain and ADG among location during the grower phase; this is in line with (Lu et al., 2007) who reported no difference between chickens exposed to different levels of temperatures in terms of BW gain.

Breed significantly influenced feed intake $(\mathrm{P}<0.05)$, these results are alike to the findings of Abiola et al. (2008) who observed that daily feed intake of chickens increased with increase in the weight of chicks and varies from breed to breed. Breed effect on feed intake among chickens raised in various systems have been also reported (Tadelle et al., 2003; Reta et al., 2012; Wondmeneh, 2015). Feed intake can be affected by many factors, but the main one is the environmental conditions such as the weather including the temperature of the region (Ferket and Gernat, 2006). This explains why the feed intake was different among location during the study period. This finding is also Supported by a report (Osti, 2017), who state a significantly different feed intake among the different climatic zones. Similar to the present study the result of Kayitesi, (2015) reported that chickens reared in Gulu district had a higher feed intake than those in Kiryandongo. This study further showed significant $(\mathrm{p}<0.5)$ difference in the effect of breed by location interaction.

Results on FCR in the present study were significantly different in the two breeds as DZ-white was significantly better in FCR than Improved Horro. The observed breed effects in affecting feed conversion ratio were in agreement with the reports of (Udeh et al., 2015). This study further showed significant difference among location. The results of the current study is in line with (Osti, 2017), who state a significantly different FCR among the different climatic zones

The results of the current study were in-line with the reports of Benyi et al.(2015), who found that mortality rate was not affected by breed. On the other hand this study result disagreed with a report of (Reta et al. 2012; Wondmeneh, 2015; Ewonetu, 2017).who found significant differences in mortality for different breed. Geographic location can influence the survival rate of chickens, this explains why location had effect on the mortality in this studies which is in-line with Aberra et al. (2013) and Alem (2014) who reported that chickens in midland of Ethiopia survive better than chickens in other agro-ecological zones. The higher mortality rate in L2 is in agreement with the findings of Azoulay et al. (2011) who found high mortality rate under hot environmental conditions. 


\section{Conclusion}

The results of this study have demonstrated that DZ-white breeds had significantly enhanced speed of weight gain as well as growth and good feed conversion ratio compared to the Improved Horro breed at the starter and grower phase. However, this study has also shown that Improved Horro chickens breeds have the genetic ability to grow fast if properly managed. The study also showed that both chicken breeds are well adapted in highland and midland agro-ecology zone and their production performance was better than the chicken kept in lowland agroecology zone. Higher mortality due to unidentified diseases could be one of the reasons for the poor production performance of the lowland agro-ecology zone observed in the current study. Generally the study indicate that significant improvement in poultry production in Ethiopia is possible through the replacement of less productive chicken breeds with these breeds and a combination of sound environmental and nutrition management.

\section{Acknowledgments}

We sincerely thank Ethiopian Institute of Agricultural Research, Wolaita Sodo and Addis Ababa Universities for their financial support. We are also thankful to all staff members of National poultry research case team at Debre Zeit, Werer Agricultural Research centers (poultry research case team), Mekelle University Department of Animal, Rangeland and Wildlife Sciences and Faji Temperate Fruits and Related Product Development at Debre Berhan for their collaboration and facilitation.

\section{References}

1) Aberra M, Zemene $\mathrm{W}$, Yosef T, 2013.Assessment of the prevailing handling and quality of eggs from scavenging indigenous chickens reared in different agro-ecological zones of Ethiopia. Journal of Environmental Occupation Sciences2(1): 1-8.

2) Abiola SS, Meshioye OO, Oyerinde BO, Bamgbose MA, 2008. Effect of Egg size on hatchability of broiler chicks. Archiv azootechnica 57: 83 -86.

3) Adedeji TA, Adebambo OA, Peter SO, Ojedapo LO, Ige AO, 2006. Growth performance of crossbreed and purebreed resulting from different sire strain in humid tropical environment. Journal of Animal and Veterinary Advances 5(8): 674-678.

4) Alabi RA, Esobhawan AO, Aruna MB 2006. Econometric determination of contribution of family poultry to women's income in Niger-delta, Nigeria. Journal of Central European Agriculture 7:753-760.

5) Alem T, 2014. Production and reproduction performance of rural poultry in lowland and midland agro-ecological zones of Central Tigray, Northern Ethiopia. British Journal of Poultry Sciences3(1): 6-14.

6) Azoulay Y, Druyan S, Yadgary L, Hadad Y, Cahaner A, 2011. The viability and performance under hot conditions of featherless broilers versus fully feathered broilers. Poultry Science 90(1): 19- 29.

7) Bekele F, Adnoy T, Gjoen HM, Kathle J, Abebe G, 2010. Production Performance of Dual Purpose Crosses of Two Indigenous with Two Exotic Chicken Breeds in Sub-tropical Environment. International Journal of Poultry Science 9: 702-710.

8) Benyi K, Tshilate TS, Netshipale AJ, Mahlak KT, 2015. Effects of genotype andsex on the growth performance and carcass characteristics of broiler chickens. Tropical Animal Health and Production 47:7:1225-1231.

9) BoPED (Bureau of Planning and Economic Development), 2011.Tigray, Ethiopia.

10) Dei HK, Alidu I, Otchere EO, Donkoh A, Boa-Amponsem K, Adam I, 2009. Improving the brooding management of local guinea fowl (Numidameleagris). Family Poultry18 (1\&2): 3-8.

11) EIAR (Ethiopian Institute of Agricultural Research), 2004.DebreBerhan Agricultural Research Center,DebreBerhan, Ethiopia.

12) Ekka R, Behura NC, Samal L, Nayak GD, Pati PK and Mishra PK 2016. Growth performance and linear body measurements of Hansli, CSML and HanslixCSML cross under intensive system of rearing. Journal of Livestock Science 7: 114-121

13) Enaiat MM, Amina AS, Eman MA, 2010. A comparative study of productive and physiological performance between two local strains of chicks. Egypt Poultry Science30: 297-316.

14) Ewonetu K, 2017.Growth performance and rearing costs of Fayoumi and White Leghor chicken breeds. Journal of East African sciences 11 (1): 37-42

15) Ferket PR, Gernat AG, 2006. Factors that affect feed intake of meat birds. International Journal of Poultry Science 5(10): 905-911.

16) Kayitesi A, 2015. Management systems and location effects on growth and carcass traits of kuroiler and local chickens MSc thesis.Makerere University, Uganda. 
17) LMP (livestock master plan), 2015. Road maps for growth and transformation. A contribution to the growth and transformation plan II (2015-2020). Ministry of Agriculture, Livestock Resources Development Sector. Addis Ababa, Ethiopia.

18) Lu Q, Wen J, Zhang H, 2007. Effect of chronic heat exposure on fat deposition and meat quality in two genetic types of chicken. Poultry Science86:1059-1064.

19) Lwelamira J, 2007. Prospects for improving performance among two local chicken ecotypes of Tanzania through selection. $\mathrm{PhD}$ thesis, SokoineUniversity of Agriculture, Morogoro, Tanzania,204 pp.

20) Mohammed MD, Abdalsalam YI, KheirA R, JinyuW, Hussein MH, 2005. Growth performance of indigenous $x$ Exotic crosses of chicken and evaluation of general and specific combining ability under Sudan condition. International Journal of Poultry Science4: 468-471.

21) Mutayoba SK, Katule AK, Minga U, Mtambo MM, Olsen JE, 2012. The effect of supplementation on the performance of free range local chickens in Tanzania. Livestock Research for Rural Development 24:5.

22) Nigussie D, Van der Waaij LH, Dessie T, Van Arendonk JAM, 2010. Production objectives and trait preferences of village poultry producers of Ethiopia: implications for designing breeding schemes utilizing indigenous chicken genetic resources. Tropical Animal Health and Production 42(7): 1519-1529.

23) Nigussie D, VanderWaaij EH, Van Arendonk JAM, 2011. Genetic and phenotypic parameter estimates for body weights and egg production in Horro chicken of Ethiopia. Tropical Animal Health and Production 43 (1): $21-28$.

24) Osti R, Bhattarai D, Zhou D, 2017. Climatic variation: effects on stress levels, feed intake, and Bodyweight of broilers. Brazilian Journal of Poultry Science 19(3): 489-496.

25) Reta D, Negussie D, Alemu Y, 2012: Comparative production performance of two exotic chicken breeds under two different feed regimes in three agro-ecologies of central Oromia, Ethiopia- a step forward for distribution or contract rearing of day old exotic chicks under rural setting. Livestock Research for Rural Development 24:article 153.

26) SAS, 2010. Statistical analysis software enterprise guide version four, SAS Institute Inc.

27) Sharma RK, 2007. Role and relevance of rural family poultry in developing countries with special reference to India. Family Poultry 17 (1\&2):35-40.

28) Sonaiya E B, Branckaert R D, Guèye E F, 1999. Research and development options for family poultry, In the scope and effect of family poultry research and development (FAO) 3-18.

29) Tadelle D, Ogle B, 2001. Village poultry production system in the Central Highlands of Ethiopia. Tropical Animal Health and Production33: 521-537.

30) Udeh I, Ezebor PN, Akporahuarbo PO, 2015. Growth performance and carcassyield of three commercial strains of broiler chickens raised in a tropical environment. Journal of Biology, Agriculture and Healthcare 2: 6267.

31) Wondmeneh E, 2015. Genetic improvement in indigenous chickens of Ethiopia: one step ahead. PhD thesis. Wageningen University, Netherland.

32) Wondmeneh E, Dawud I, Alemayehu A, Meskerem A, Tadios H, 2012. Enhancing the genetic basis of commercial layer industry through introduction and evaluation of dual-purpose chicken breed (Potchefstroom Koekoek breed). Ethiopian Society of Animal production (ESAP). Addis Ababa, Ethiopia.

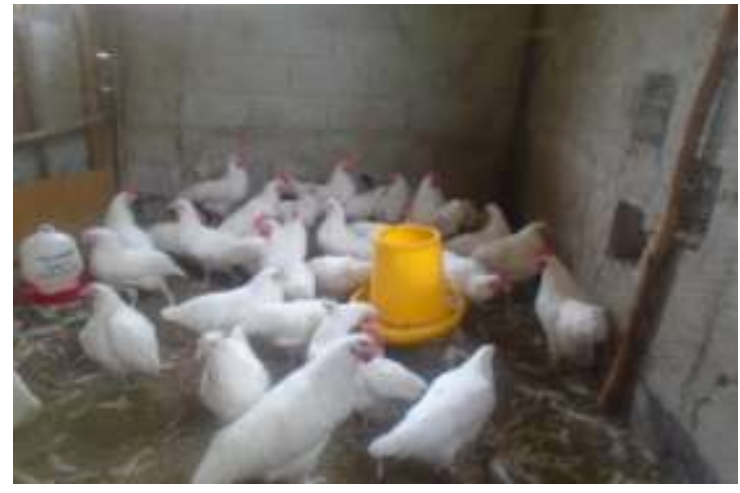

DZ-white chicken

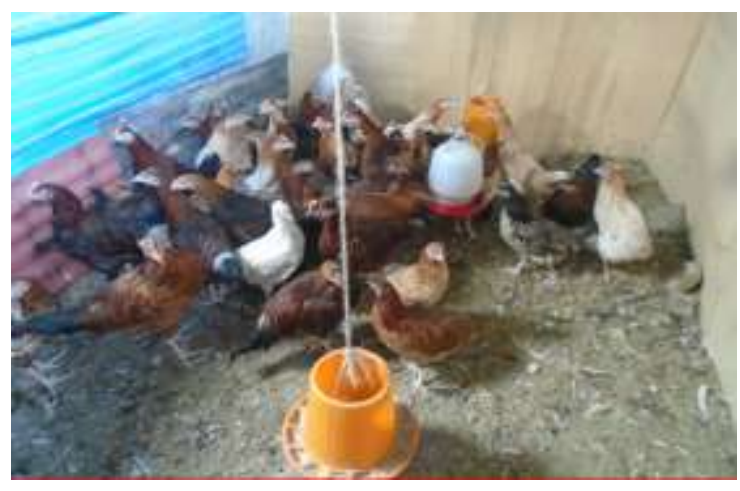

Improved Horro chicken 